\title{
Evaluación de un sistema de muestreo pasivo de CPVC para dióxido de nitrógeno en el aire
}

\author{
Omar Rojas-Bolaños' \\ Irene Jiménez-Barrantes ${ }^{2}$
}

Fecha de recepción: 10 de febrero del 2011

Fecha de aprobación: 23 de julio del 2011

Rojas, O; Jiménez, I. Evaluación de un sistema de muestreo pasivo de CPVC para dióxido de nitrógeno en el aire. Tecnología en Marcha. Vol. 25, NN I. Enero-Marzo 2012 Pág 44-53

I. Químico. Laboratorio de Calidad de Aire. Centro de Investigación en Contaminación Ambiental, Universidad de Costa Rica. Teléfono: 88498281.Correo electrónico: omar.rojas@ucr.ac.cr

2. Química. Escuela de Química, Universidad de Costa Rica. San José, Costa Rica. Teléfono: 25 I I-5376.

Correo electrónico: irene.jimenez@ucr.ac.cr 


\section{Palabras clave}

Contaminación del aire, muestreo activo, muestreo pasivo, tubos de difusión pasiva, dióxido de nitrógeno, óxido nítrico, ozono, CPVC.

\section{Resumen}

Se construyeron tubos de difusión pasiva para determinar la concentración de $\mathrm{NO}_{2}$ en el aire con tubos de poli(cloruro de vinilo) clorado (CPVC). Su desempeño se evaluó durante el 2000, por medio de comparaciones de sus resultados con los resultados obtenidos al utilizar el método modificado de Griess-Saltzman, recomendado por la Organización Mundial de la Salud.

Los resultados de la comparación mostraron una buena concordancia entre ambas metodologías con respecto a las concentraciones de $\mathrm{NO}_{2}$ de 45-85 $\mu \mathrm{g} / \mathrm{m}^{3}$. Al utilizar la regresión lineal por mínimos cuadrados simples y la prueba de rangos y signos de Wilcoxon, no se encontró diferencia significativa entre los resultados aportados por ambos métodos. El sesgo relativo calculado en 7 comparaciones varió de $-19,05 \%$ a $24,44 \%$ con un promedio de $5,76 \%$.

Los resultados indicaron que los tubos pasivos sobreestimaron la concentración de $\mathrm{NO}_{2}$, sin embargo, este sesgo no fue superior a los reportados por otros autores.

La precisión del sistema pasivo, medida en cuatro tubos por medio del coeficiente de variación, estuvo en el ámbito de 5,2\% a 10,6\%, y es similar a la reportada por otros investigadores.

\section{Key words}

Air pollution, active sampling, passive sampling, passive diffusion tubes, nitrogen dioxide, nitric oxide, ozone, CPVC.

\begin{abstract}
Passive diffusion tubes were made to detect $\mathrm{NO}_{2}$ concentration in the air with chlorinated polyvinyl chloride's tubes (CPVC). Evaluation was carried out by comparing the results obtained in 2008 with the Griess-Saltzman modified method, according to World Health Organization guidelines.

Statistical analysis done with linear minimal squares and Wilcoxon ranges and signs found no significant difference between both methods. Relative bias in 7 samples varied from $-19.05 \%$ to $24.44 \%$ with an average value of $5.76 \%$.
\end{abstract}

Results indicated that the passive tubes overestimated the concentration of $\mathrm{NO}_{2}$, however, this bias was not greater than the ones reported by other authors. The passive system's precision, measured by variation coefficient in a four tube sample, was in the $5.2 \%$ to $10.6 \%$ range, again, similar to other reported ranges. 


\section{Introducción}

Los dispositivos de muestreo pasivo para especies químicas en la atmósfera son capaces de recolectar muestras sin forzar el movimiento del aire a través del instrumento por medio de una bomba de vacío u otro mecanismo (UNEP/WHO, 1994).

Los primeros sistemas pasivos fueron desarrollados por Palmes, Gunnison, DiMattio y Tomczyk (1976), cuyo diseño consistió de un tubo cilíndrico cerrado en uno de sus extremos, en donde se colocaba un cedazo impregnado con una disolución capaz de retener a la especie química cuya concentración se deseaba conocer. Aunque hoy en día existen otros diseños, el sistema mencionado sigue en uso (Vardoulakis, Lumbreras y Solazzo, 2009).

Su funcionamiento se basa en la difusión molecular que ocurre de una región de alta concentración (extremo abierto del tubo), hacia otra de baja concentración (extremo cerrado del tubo), fenómeno gobernado por la primera ley de Fick (Plaisance, Sagnier, Saison, Galloo, Guillermo, 2002). Si se aplica dicha ley a un cilindro con un área de sección transversal (a) expresada en metros cuadrados, longitud (I) en metros, y si se considera que la concentración del analito en el extremo cerrado del tubo es cero, es posible obtener una expresión que permita calcular la concentración (C) del contaminante en el extremo abierto del tubo.

$$
C=\frac{Q l}{D_{1,2} a t}
$$

En la expresión anterior, (C) es la concentración de $\mathrm{NO}_{2}$ en el aire medida en $\mu \mathrm{g} / \mathrm{m}^{3}$, Q es la masa, en $\mu \mathrm{g}$, del gas de interés transferida a lo largo del tubo en $t$ segundos, y $D_{1,2}$ es el coeficiente de difusión del gas en el aire. Dicho parámetro puede ser determinado experimentalmente $\mathrm{u}$ obtenido de la literatura (UNEP/MHO, 1994).

Por su bajo costo (Namiesnik, Zabiegala, Kot-Wasik, Partyka y Wasik, 2005) y la sencillez de los métodos analíticos involucrados (Cape, 2009), este tipo de dispositivos se presenta como una opción atractiva para llevar a cabo estudios y programas de evaluación de la calidad del aire tanto en zonas rurales como urbanas, ya que permite obtener información de manera económica, fiable y continua.
Con respecto a los materiales utilizados para la construcción de los tubos pasivos, se ha reportado el uso de polietileno (Kasper-Giebl y Puxbaum, 1999), cuarzo, papel (Heal, O'Donoghue y Cape, 1999). y acrílicos (Vardoulakis et al, 2009). El poli(cloruro de vinilo) clorado se presenta como una opción interesante por su bajo costo, resistencia, amplia disponibilidad en el mercado costarricense y opacidad. Esta última característica es requerida para evitar la fotólisis del $\mathrm{NO}_{2}$ en el interior del tubo (Kirby, Fox, Waterhouse y Drye, 200 I).

En la presente investigación se muestran los resultados obtenidos en la evaluación de un sistema de muestreo pasivo de dióxido de nitrógeno en el aire, construido con poli(cloruro de vinilo) clorado (CPVC), al compararlo con un método activo recomendado por la Organización Mundial de la Salud (UNEP/NHO, 1994).

\section{Metodología}

En este trabajo se utilizó reactivos calidad para un análisis sin purificación previa, y todas las disoluciones se prepararon con agua de alta resistividad $(>18$ $\mathrm{M} \mathrm{cm})$.

Las disoluciones madre de nitrito de sodio se prepararon con $\mathrm{NaNO}_{2}$ sólido (99,0\% puro, marca Merck, artículo 6549 del lote 00IKI320I449) desecado a $110^{\circ} \mathrm{C}$ durante I hora. Se midió una masa de 0,2000 g del reactivo, se disolvió y se diluyó a 200,0 ml con agua desionizada. La disolución se guardó en una botella oscura de polietileno de alta densidad en refrigeración durante un tiempo máximo de tres meses.

La disolución generadora de color, conocida como disolución de Saltzman, se preparó según las indicaciones de Yamada, Kimura, Tomozawa y Fuse (1999). Se disolvió 5,0 g de ácido sulfanílico en 700 $\mathrm{ml}$ de agua desionizada, se agregó $50 \mathrm{ml}$ de ácido fosfórico concentrado y $50 \mathrm{ml}$ de una disolución acuosa de clorhidrato de $\mathrm{N}$ - ( I - naftil) etilendiamina al 0,1\% (porcentaje en masa) y se diluyó a I,0 I. Por último se guardó en una botella oscura de polietileno de alta densidad y en refrigeración.

Las mediciones espectrofotométricas se realizaron con un espectrofotómetro SHIMADZU UV - 1203. 

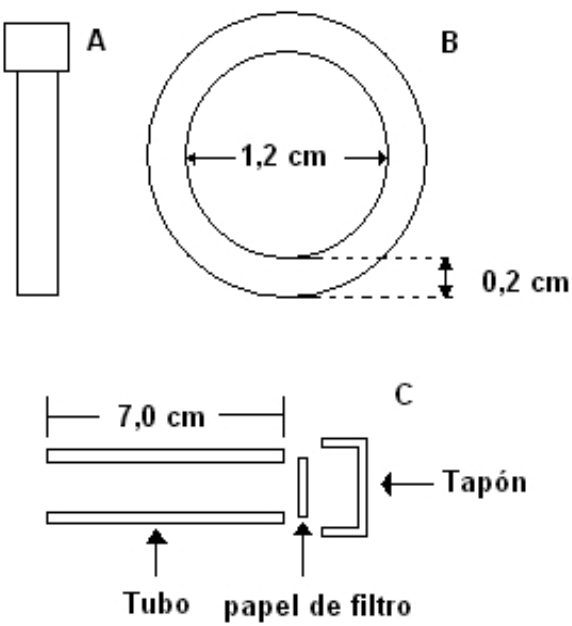

Figura ।. Diseño del sistema pasivo construido: A) tubo pasivo ensamblado, B) sección transversal del tubo utilizado, C) ensamblaje del sistema pasivo.

Los datos de temperatura y presión atmosférica, fueron registrados cada hora por medio de una mini estación meteorológica Nielsen-Kellerman modelo Kestrel 4000.

\section{Construcción de los sistemas pasivos y recolección de las muestras}

Los dispositivos de muestreo pasivo construidos fueron del tipo tubo (figura IA), basados en el diseño de Palmes, Gunnison, DiMattio y Tomczyk (1976).

Los sistemas pasivos se construyeron con tubos de CPVC de 1,2 cm de diámetro interno y con un espesor de pared de 0,2 cm (figura IB). De este tubo se cortaron cinco piezas de 7,0 cm de longitud. El largo y el diámetro interno de cada tubo fueron medidos con un vernier. Por cada tubo cortado se adquirió un tapón de CPVC del mismo diámetro externo del tubo.

Los tubos y tapones fueron lavados con agua y detergente para cristalería. Se dejaron en agua jabonosa por 24 horas y se enjuagaron con agua desionizada, luego se colocaron dentro de un beaker de I I y se cubrieron con más agua desionizada. Posteriormente, se colocó el beaker dentro de un baño ultrasónico por I hora. Transcurrido este tiempo, se sacaron los tubos y tapones del beaker y se les permitió secarse al aire.

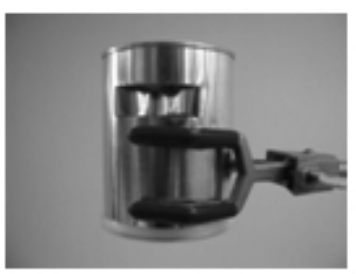

A

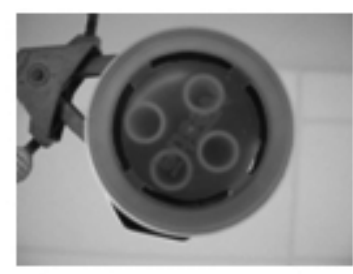

B

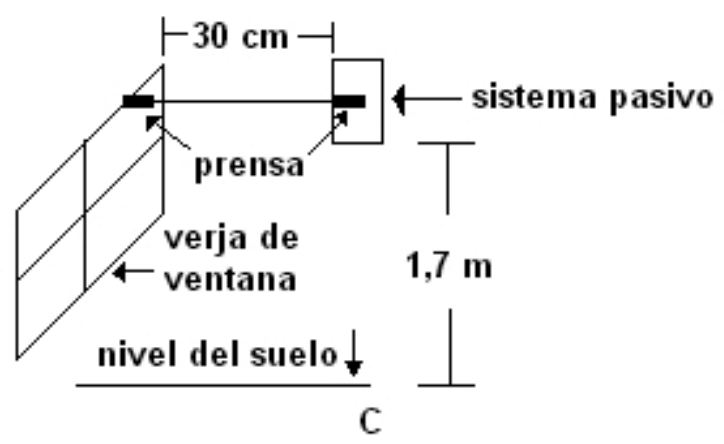

Figura 2. A) Vista lateral del sistema completo, B) vista transversal del sistema completo, C) instalación del sistema en el campo.

En uno de los extremos de cada tubo se dispuso un filtro de celulosa (ALBET I45) impregnado con una disolución acuosa de trietanolamina al 20\% (porcentaje en masa). Posteriormente, se colocó un tapón de CPVC sobre el extremo que soportaba al filtro (figura IC) y se tapó el extremo abierto de los tubos con papel Parafilm $M \circledR$. Se introdujeron cuatro tubos dentro de un recipiente previamente perforado en su costado y base, de tal forma que el viento no diera directamente en las entradas de los tubos (figura 2A y 2B).

Posteriormente se guardó el recipiente con los tubos dentro de una bolsa plástica con cierre hermético hasta su traslado al sitio de muestreo con el fin de reducir las posibilidades de contaminación. El quinto tubo no fue expuesto al aire ambiente durante todo el periodo de muestreo y se identificó como muestra blanco.

En el sitio de muestreo se sacó el sistema pasivo de la bolsa y se instaló a 1,7 m de altura sobre el nivel del suelo por medio de una prensa universal unida a la verja de una de las ventanas (figura 2C), se retiraron los papeles que cubrían los extremos abiertos de los tubos y se dejaron en el lugar por un periodo de 10 a 15 días. 


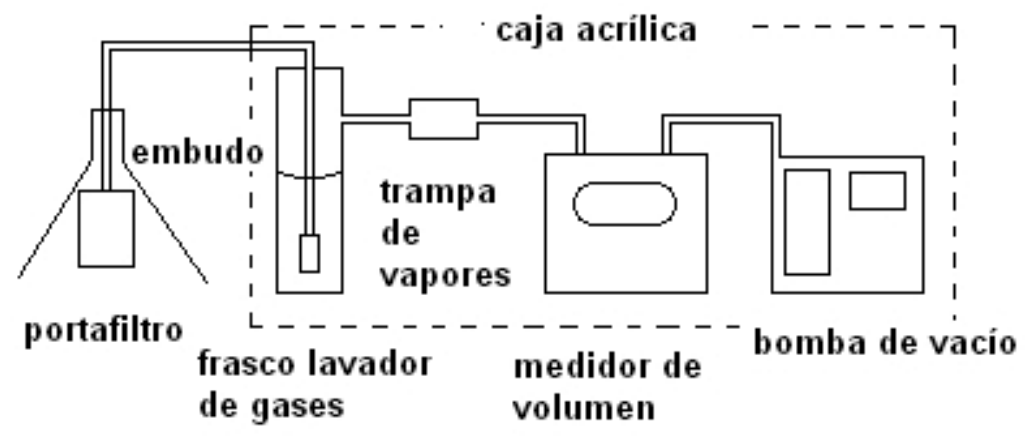

Figura 3. Esquema del equipo de muestreo activo utilizado en la evaluación de los sistemas pasivos.

Una vez finalizado el periodo de exposición de los tubos pasivos, se trasladaron hasta el laboratorio dentro de una bolsa plástica.

\section{Sistema activo}

La evaluación del desempeño del sistema pasivo se llevó a cabo por medio de una comparación con un sistema de muestreo activo no automático basado en el método español modificado de GriessSaltzman, cuyo esquema se muestra en la figura 3 (UNEP/WHO, 1994).

El sistema de la figura está constituido por un portafiltros NILU (Instituto Noruego de Investigación del Aire) diseñado para filtros de $37 \mathrm{~cm}$ de diámetro. En este, se colocó un filtro de nitrato de celulosa con un tamaño de poro de 0,4 $\mu \mathrm{m}$, con el fin de evitar que las partículas suspendidas en el aire llegaran a la disolución absorbente. Esta última se colocó en un frasco lavador de gases dotado con un vidrio poroso para aumentar la superficie de contacto entre la muestra de aire y la disolución.

Seguidamente, se ensambló una trampa rellena con silica gel para evitar que los vapores de la disolución alcanzaran al resto de los componentes. A continuación se colocó un medidor de volumen de aire GALLUS 2000 previamente verificado con un medidor de flujo trazable al NIST, marca BIOS DryCal DC-Lite modelo ML (certificado de calibración No. I850 I).

La succión de la muestra se llevó a cabo con una bomba de vacío marca SKC modelo 224-PCXR8, modificada para funcionar con una batería de motocicleta de 6 voltios con el fin de hacer muestreos de 24 horas por cada carga de batería. En pruebas posteriores, la batería de motocicleta fue sustituida por un sistema de potencia continuo (UPS) y un transformador con el fin de asegurar el funcionamiento de la bomba de muestreo por periodos de tiempo mayores. El portafiltros se colocó dentro de un embudo plástico para protegerlo de la lluvia y el resto de los componentes se ensamblaron dentro de una caja acrílica como medida de protección. Como absorbente se utilizaron 50,00 $\mathrm{ml}$ de una disolución acuosa de trietanolamina al 3,0\% (porcentaje en masa). Se utilizaron flujos de muestreo de $0,15 \mathrm{l} / \mathrm{min}$.

La eficiencia de la recolección se evaluó al conectar dos frascos lavadores de gases en serie y al realizar el análisis en cada disolución. En todas las pruebas no se detectó nitrito en el segundo frasco. El tiempo de muestreo con el sistema activo varió de dos a tres días. En otras pruebas se verificó que las muestras líquidas se mantuvieron estables por cuatro días sin necesidad de someterlas a refrigeración.

\section{Sitio de muestreo}

Los muestreos se realizaron en el jardín del Centro de Investigación en Estudios de la Mujer (CIEM), ubicado en la sede Rodrigo Facio de la Universidad de Costa Rica. Es una zona urbano-comercial, cercana a una calle angosta de dos carriles y con alto flujo vehicular en ambos sentidos. A menos de $50 \mathrm{~m}$ se encuentra la llamada "Rotonda de Betania", que es un punto común para muchos conductores que se dirigen día a día a diferentes puntos de la capital.

Análisis de las muestras

Las muestras se cuantificaron al intercalarlas en una curva de calibración preparada a partir de la disolución madre de $\mathrm{NaNO}_{2}$ de acuerdo con el 
siguiente procedimiento: se preparó una disolución intermedia diluyendo $125 \mu \mathrm{l}$ de la disolución madre a 25,00 $\mathrm{ml}$ con agua desionizada. Se tomó I00, 200, 250, 300, 400, 500 y $600 \mu \mathrm{l}$ de la disolución anterior. Cada alícuota se vertió en un matraz aforado de I0,00 ml y se aforó con la disolución de Saltzman. Se esperó 30 minutos hasta que la coloración fucsia se estabilizara y se midió la absorbancia de cada disolución a $540 \mathrm{~nm}$.

Se desarmaron los tubos pasivos correspondientes a las muestras recolectadas y a la muestra blanco. Cada papel de filtro se colocó dentro de un beaker de $50 \mathrm{ml}$ y a cada uno se le adicionó $10,00 \mathrm{ml}$ de la disolución de Saltzman. Se taparon los beakers con vidrios de reloj y se mantuvieron en la oscuridad por I hora. Las disoluciones adquirieron una coloración fucsia.

Alícuotas de 2,00 a 5,00 ml de las muestras recolectadas con el método activo se diluyeron hasta 10,00 mL con la disolución de Saltzman.

Se midieron las absorbancias a $540 \mathrm{~nm}$ de las disoluciones de las muestras pasivas y activas. Se interpolaron las absorbancias registradas en la curva de calibración tomando en cuenta que I mol de $\mathrm{NO}_{2}$ tiene la misma absorbancia que 0,74 moles de $\mathrm{NaNO}_{2}$ (Saltzman, 1954; Scaringelli, Rosenberg y Rehme, 1970; World Health Organization, 1976).

En todos los análisis realizados, la absorbancia de la disolución de la muestra blanco fue menor que la absorbancia de la disolución patrón más diluída.

Cálculo de la concentración de $\mathrm{NO}_{2}$ en el aire mediante el sistema pasivo

La concentración de $\mathrm{NO}_{2}$ en el aire se calculó, para cada uno de los cuatro tubos dentro del sistema pasivo, gracias a la ecuación I. Como coeficiente de difusión del $\mathrm{NO}_{2}$ en aire, se utilizó el valor de $0,0000154 \mathrm{~m}^{2} \mathrm{~s}^{-1}$. Este valor fue reportado por Glasius, Funch, Stroyer y Lohse (1999), quienes describieron su dependencia con la temperatura. Durante el transcurso del proyecto, se verificó que, para las temperaturas de muestreo registradas, las variaciones de este coeficiente fueron insignificantes.

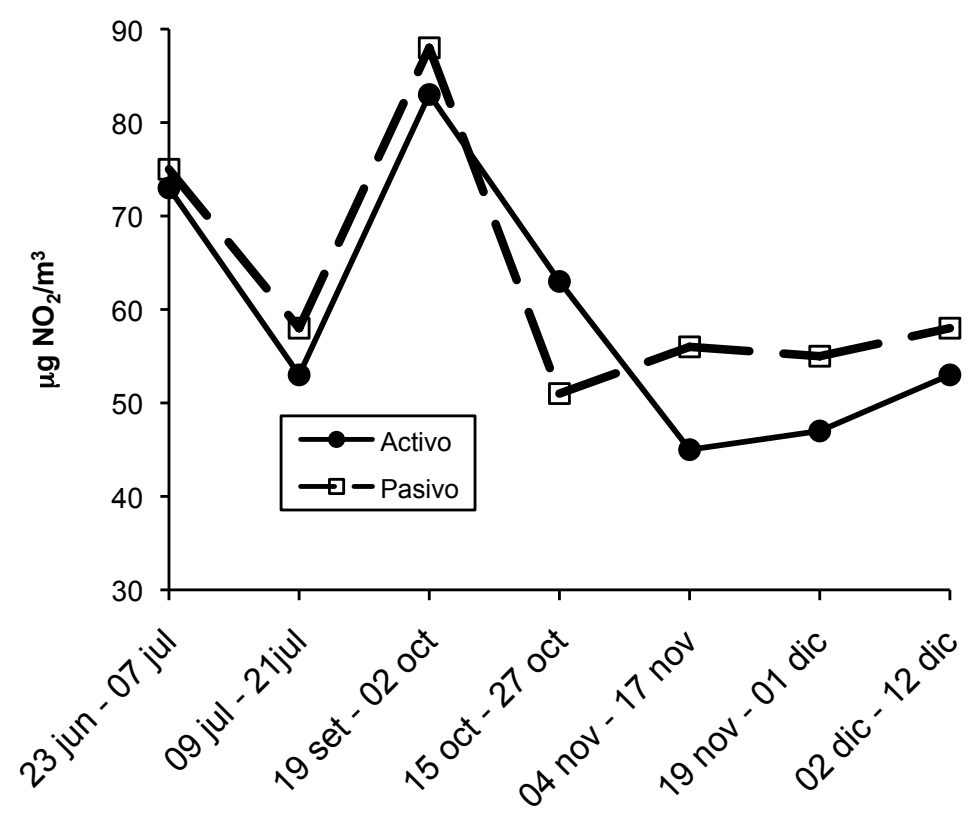

Figura 4. Concentraciones de $\mathrm{NO}_{2}$ en aire obtenidas de manera simultánea por las dos metodologías utilizadas durante el 2008. 
Al promediar las concentraciones de $\mathrm{NO}_{2}$ en cada tubo, se consiguió la concentración final de $\mathrm{NO}_{2}$ en el aire durante el periodo de muestreo.

Cálculo de la concentración de $\mathrm{NO}_{2}$ en el aire utilizando el sistema activo

Para obtener la concentración en $\mu \mathrm{g} / \mathrm{m}^{3}$ de $\mathrm{NO}_{2}$ en el aire por medio del sistema activo, se dividieron los microgramos de $\mathrm{NO}_{2}$ obtenidos en la interpolación de cada muestra, entre el volumen de aire muestreado y expresado a $25^{\circ} \mathrm{C}$ y $101325 \mathrm{~Pa}$.

\section{Evaluación del sistema pasivo}

Ambos sistemas (pasivo y activo) se instalaron a un metro de distancia uno del otro en el sitio de muestreo durante el 2008. Los dos equipos funcionaron de manera simultánea. Por cada periodo de muestreo del sistema pasivo, se recolectaron 5 muestras con el sistema activo. Se calculó el promedio de los resultados obtenidos de las muestras activas y se comparó con el promedio de los tubos pasivos.
La exactitud del sistema pasivo se evaluó por medio del sesgo relativo (SR) definido por la siguiente expresión:

$$
S R=\frac{(C p-C a)}{C a} * 100
$$

En esta ecuación, $\mathrm{Cp}$ es la concentración de $\mathrm{NO}_{2}$ en el aire medida con el sistema pasivo, y Ca es la concentración de $\mathrm{NO}_{2}$ en el aire medida con el sistema activo (Vardoulakis, Lumbreras y Solazzo, 2009).

Se utilizó una regresión lineal por mínimos cuadrados simples y la prueba no paramétrica de rangos y signos de Wilcoxon como criterios de comparación analítica entre los métodos (Miller y Miller, 2002).

La precisión del sistema pasivo se evaluó por medio del coeficiente de variación (CV) (Miller y Miller, 2002), definido por la siguiente expresión:

$$
C V=\frac{s}{x} * 100
$$

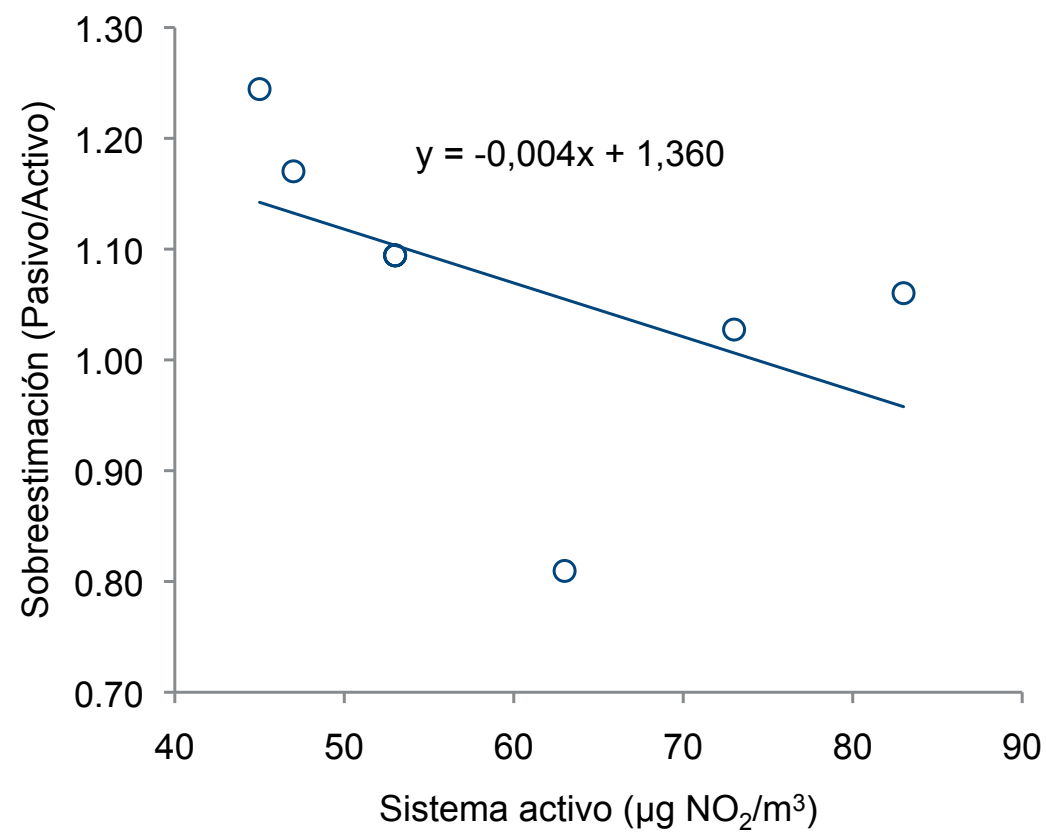

Figura 5. Relación pasivo/activo y concentración del sistema activo. En la gráfica se muestran 6 puntos debido a que los resultados de dos comparaciones fueron idénticos. 
Para este estudio, s es la desviación estándar de las concentraciones de $\mathrm{NO}_{2}$ obtenidas por los cuatro tubos dentro del sistema pasivo, $y x$ es la concentración promedio del $\mathrm{NO}_{2}$ calculada para los mismos cuatro tubos.

\section{Resultados y discusión}

\section{Uso del sistema pasivo}

El uso de ambas metodologías dejó en evidencia la simplicidad del sistema pasivo. La ausencia de frascos lavadores de gases, medidores de volumen y bombas de vacío eléctricas facilitó, en gran medida, la recolección de muestras en el campo, así como su transporte al laboratorio. Debido a que no se utilizó una disolución como medio absorbente, no hubo problemas por efecto de la evaporación. La preparación de los tubos una vez cortados y lavados no requirió más de 15 minutos. El tiempo invertido en el análisis de laboratorio fue similar al utilizado en el método activo (aproximadamente una hora).

\section{Exactitud de los sistemas pasivos}

Las concentraciones de $\mathrm{NO}_{2}$ en el aire medidas por medio de tubos pasivos, mostraron buena concordancia con las concentraciones obtenidas a partir del sistema activo (figura 4).

El sesgo relativo en cada comparación varió desde $-19,05 \%$ hasta $24,44 \%$, con un valor promedio de $5,76 \%$.

Los resultados de la comparación de ambas metodologías por medio de una regresión lineal obtenida a partir el método de mínimos cuadrados simples (Miller y Miller. 2002) se muestran en el cuadro I.

\section{Cuadro I. Resultados de la regresión lineal entre la metodología pasiva y activa}

\begin{tabular}{|c|c|}
\hline No de observaciones & 7 \\
\hline Pendiente & $0,8 \mid \pm 0,56$ \\
\hline Intercepto & $\mid 4,56 \pm 34,13$ \\
\hline Coeficiente de correlación $r$ & 0,8580 \\
\hline Diferencia significativa & No \\
\hline
\end{tabular}

Los valores de la pendiente y el intercepto, junto con sus límites de confianza calculados para un nivel de significación $\mathrm{P}=0,05$, indican que no existe diferencia significativa entre los resultados obtenidos por ambas metodologías.

A partir de la prueba no paramétrica de rangos y signos de Wilcoxon (Miller y Miller, 2002), se obtuvo un resultado similar (cuadro 2).

Cuadro 2. Resumen de la prueba de rangos y signos de Wilcoxon

\begin{tabular}{|c|c|}
\hline Estadístico de la prueba & 7 \\
\hline Valor crítico tabulado $(\mathrm{P}=0,05)$ & 2 \\
\hline ¿Se rechaza hipótesis nula? & No \\
\hline
\end{tabular}

Debido a que el estadístico de la prueba es mayor que el valor crítico tabulado, se debe aceptar la hipótesis nula: no hay evidencia de que la media de las diferencias de las concentraciones aportadas por ambos métodos sea significativamente diferente de cero, por lo que no hay diferencia sistemática entre los métodos analíticos bajo las condiciones de evaluación.

En la figura 5 se aprecia que el método pasivo tiende a sobreestimar la concentración de $\mathrm{NO}_{2}$ medida con el sistema activo ( 6 de 7 puntos por encima de $y=1$ ). Esta sobrestimación fue discutida por Heal y Cape (1997), quienes la atribuyeron a la oxidación del NO por el $\mathrm{O}_{3}$ en el interior del tubo, lo que produjo una mayor concentración de $\mathrm{NO}_{2}$. Por medio de un modelo numérico, los mismos autores predijeron un sesgo relativo del 28\% para zonas urbanas. Este valor es superior al sesgo promedio obtenido, pero se aproxima al sesgo máximo calculado en las comparaciones individuales (24,44\%). Los resultados contradicen lo reportado por Vardoulakis et al. (2009), quienes concluyeron que los tubos pasivos subestiman la concentración de $\mathrm{NO}_{2}$ con un SR máximo de -7,61 \%.

La correlación negativa de la gráfica en la figura 5 indica que la sobreestimación se hace menos importante al aumentar la concentración del $\mathrm{NO}_{2}$ en el aire. Heal, O'Donoghue y Cape (1999) atribuyeron este fenómeno a dos factores que, en conjunto, tienden a reducir el efecto de la reacción entre el óxido nítrico y el ozono en el interior del 
tubo: a) la disminución de la concentración de $\mathrm{NO}$ a elevadas concentraciones de $\mathrm{NO}_{2}$ y b) la disminución del $\mathrm{O}_{3}$ troposférico a elevadas concentraciones de NOx. Estos factores favorecen el uso de los sistemas pasivos en zonas rurales, debido, principalmente, a la baja densidad vehicular y a la ausencia de fuentes emisoras estacionarias, lo que dio como resultado bajos niveles de óxido nítrico en el aire. Vardoulakis et al. (2009) reportaron un menor sesgo al lado de caminos rurales que en zonas urbanas, lo que apoya esta aseveración.

La fluctuación de valores mostrados en el eje de las ordenadas de la figura 5 imposibilita utilizar un factor de corrección que permita ajustar los resultados de los tubos de difusión a las concentraciones obtenidas por el método de referencia. Una afirmación similar fue reportada por Heal, et al. (1999).

\section{Precisión de los sistemas pasivos}

Los coeficientes de variación calculados para diferentes periodos de medición a lo largo del 2008 se muestran en el cuadro 3.

Cuadro 3. Coeficientes de variación calculados para los sistemas pasivos

\begin{tabular}{|c|c|}
\hline Periodo & CV \\
\hline $7-29$ enero & 5,2 \\
\hline 13 febrero -12 marzo & 8,1 \\
\hline 23 junio -7 julio & 10,5 \\
\hline $9-21$ julio & 6,7 \\
\hline 19 setiembre -2 octubre & 1,9 \\
\hline 15 octubre -27 octubre & 10,6 \\
\hline 4 noviembre -17 noviembre & 7,6 \\
\hline 19 noviembre -1 diciembre & 9,0 \\
\hline 2 diciembre -12 diciembre & 7,8 \\
\hline CV promedio & 7,5 \\
\hline
\end{tabular}

Los coeficientes de variación son similares a los reportados por Vardoulakis, et al. (2009), quienes calcularon, para sistemas de tres tubos pasivos, coeficientes de variación desde 0,3\% hasta 15,7\%, con un CV promedio de 5\%. La precisión reportada por Bush, Smith, Stevenson y Moorcroft (200I) para mediciones realizadas con dos tubos pasivos es inferior a la del cuadro 3 con un intervalo de 0,7 a $34,7 \%$ y un valor promedio de 10,3\%. Gair, Penkett y Oyola (|99|) reportaron un CV promedio de 6,5\%, mientras que en la revisión hecha por Moon (200 I) se indica que la precisión de los tubos pasivos es buena y se encuentra en el rango de $5 \%$ a $10 \%$.

\section{Conclusiones}

La evaluación de los sistemas pasivos construidos demostró que su desempeño fue similar al reportado por otros autores. Se encontró que los tubos tienden a sobreestimar la concentración de $\mathrm{NO}_{2}$ en el aire medida con el sistema activo. Esta desviación puede ser despreciada ya que se ve compensada por su bajo precio y versatilidad. Los resultados indicaron que el uso de CPVC no afecta el desempeño del tubo pasivo.

\section{Agradecimientos}

Agradecemos a la Vicerrectoría de Investigación de la Universidad de Costa Rica por el apoyo financiero brindado a la presente investigación a través del proyecto $N^{\circ}$ 802-A6-127. De la misma forma, agradecemos al personal del Centro de Investigación en Contaminación Ambiental por brindarnos el espacio y equipos para llevar a cabo el presente trabajo.

Nuestro especial agradecimiento a las funcionarias del Centro de Investigación en Estudios de la Mujer quienes, con mucha amabilidad, nos permitieron realizar las mediciones de campo en sus instalaciones.

\section{Bibliografía}

Bush, T; Smith, S; Stevenson, K \& Moorcroft, S. (200 I). Validation of Nitrogen Dioxide Diffusion Tube Methodology in the UK, Atmos. Environ. 35, 289-296.

Cape, J. N. (2009). The use of passive diffusion tubes for measuring concentrations of nitrogen dioxide in air. Critical Reviews in Analytical Chemistry. 39, 289-3 10.

Decreto No 3022I-S. (2002, 21 de marzo) Reglamento sobre inmisión de contaminantes atmosféricos. La Gaceta (54) 25 a la Gaceta No. 57 del jueves 21.03.2002).

Gair, A. J; Penkett, S. A. \& Oyola, P. (|99|). Development of a simple passive technique for the determination of nitrogen dioxide in remote continental locations. Atmos. Environ, 25 A, 1927-1939.

Glasius, M; Funch, M; Stroyer, T. \& Lohse, C. (1999). Measurements of nitrogen dioxide on Funen using diffusion tubes. Atmospheric Environment, 33. I I77-1 I 85. 
Heal, M. R \& Cape, J. N. (1997). A numerical evaluation of chemical interferences in the measurement of ambient nitrogen dioxide by passive diffusion samplers. Atmospheric Environment, 31, 191 I-1923.

Heal, M. R., O'Donoghue, M. A; Cape, J. N. (1999). Overestimation of urban nitrogen dioxide by passive diffusion tubes: a comparative exposure and model study. Atmospheric Environment, 33, 513-524.

Kasper-Giebl, A. \& Puxbaum, H. (1999). Deposition of particulate matter in diffusion tube samplers for the determination of $\mathrm{NO}_{2}$ and $\mathrm{SO}_{2}$. Atmospheric Environment, 33(8), | 323-1326.

Kirby, C; Fox, M; Waterhouse, J. \& Drye,T. (200I). Influence of environmental parameters on the accuracy of nitrogen dioxide passive diffusion tubes for ambient measurement. Journal of Environmental Monitoring, 3, I50- 158.

Miller, N. J. \& Miller, C. J. (2002). Estadística y quimiometría para química analítica. Madrid: Pearson Education, S. A.

Monn, C. (200I). Exposure Assessment of Air Pollutants: A Review on Spatial Heterogeneity and Indoor/Outdoorl Personal Exposure to Suspended Particulate Matter, Nitrogen Dioxide and Ozone. Atmos. Environ, 35, I-32.

Namiesnik, J; Zabiegala, B; Kot-Wasik, A; Partyka, M. \& Wasik, A. (2005). Passive sampling and/or extraction techniques in environmental analysis: a review. Analytical and Bioanalytical Chemistry, 381, 279-301.

Palmes, E. D; Gunnison, A. F; DiMattio, J. \& Tomczyk, C. (1976). Personal sampler for nitrogen dioxide. Am. Ind. Hyg. Assoc, J. 37, 570-577.
Plaisance, H; Sagnier, J; Saison, J. Y; Galloo, J. C. \& Guillermo, R. (2002). Performances and Application of Passive Sampling Method for the Simultaneous Determination of Nitrogen Dioxide and Sulfur Dioxide in Ambient Air. Environmental Monitoring and Assessment. 79, 30 I-3I 5.

Saltzman, B. E. ( 1 954). Colorimetric microdetermination of nitrogen dioxide in the atmosphere. Analytical Chemistry, 26 (12), 1949-1955.

Scaringelli, F. P; Rosenberg, E. \& Rehme, K. A. (1970). Comparasion of Permeation Devices and Nitrite ion as standards for the colorimetric determination of nitrogen dioxide. Environmental Science and Technology, 4 (I I), 924-929.

UNEP/WHO. (1994). GEMS/AIR Methodology Reviews Vol. 4: Passive and Active Sampling Methodologies for Measurement of Air Quality. WHO/EOS/94.4, UNEP/ GEMS/94.A.5. UNEP Nairobi.

Vardoulakis, S; Lumbreras, J. \& Solazzo, E. (2009). Comparative evaluation of nitrogen oxides and ozone passive diffusion tubes for exposure studies. Atmospheric Environment, 43, 2509-2517.

World Health Organization. (1976). Selected Methods of measuring air pollutants. Geneva.

Yamada, E; Kimura, M;Tomozawa, K. \& Fuse,Y. (1999). Simple Analysis of Atmospheric $\mathrm{NO}_{2}, \mathrm{SO}_{2}$, and $\mathrm{O}_{3}$ in Mountains by Using Passive Samplers. Environmental Science \& Tecnology, 33, 4| 4 |-4| 45. 
Tecnología en Marcha,

54 Vol. 25, N. ${ }^{\circ}$ I, Enero-Marzo 2012 